\title{
Influenza virus non-structural protein 1 inhibits the production of interferon $\beta$ of alveolar epithelial cells upon the infection of influenza A H1N1
}

\author{
HAO JIANG ${ }^{1,2}$, SI-MEI SHEN ${ }^{1}$, JIE YIN $^{1}$, PENG-PENG ZHANG $^{1}$ and YI SHI $^{1}$ \\ ${ }^{1}$ Department of Respiratory and Critical Care Medicine, Jinling Hospital, Nanjing University School of Medicine, \\ Nanjing, Jiangsu 210002; ${ }^{2}$ Department of Emergency Medicine, The Second Affiliated Hospital, \\ Southeast University, Nanjing, Jiangsu 210003, P.R. China
}

Received March 1, 2017; Accepted July 12, 2017

DOI: $10.3892 / \mathrm{mmr} .2017 .7138$

\begin{abstract}
Influenza A affects a large population worldwide. Influenza virus evades immune responses via various mechanisms, including through the modification of the immune microenvironment. Influenza virus non-structural protein 1 (NS1) encoded by the virus genome inhibits type I interferon (IFN) signaling pathways, which is essential for viral clearance. However, the precise mechanisms of NS1-mediated immune suppression remain poorly understood. The results of the present study demonstrated that mice infected with NS1-expressing influenza A H1N1 virus had lower expression levels of IFN $\beta$ in the lung. In addition, it was revealed that the human alveolar epithelial A549 cell line infected with influenza virus A H1N1 produced antiviral IFN $\beta$. The production of IFN $\beta$ during infection was demonstrated to be a self-dependent autocrine process. A549 cells transfected with H1N1 NS1 lost the ability to produce IFN $\beta$ upon H1N1 infection or IFN $\beta$ stimulation. NS1 inhibited the expression of IFN receptors. Furthermore, NS1 inhibited the activation of signal transducers and activators of transcription (STAT)1 and STAT2, as well as the consequent IFN $\beta$ production. These data indicate that NS1 serves an important role during virus evasion by affecting the production of IFN $\beta$ via multiple mechanisms.
\end{abstract}

\section{Introduction}

Influenza viruses are a class of highly contagious pathogen affecting both human and animals, which result in seasonal

Correspondence to: Dr Yi Shi, Department of Respiratory and Critical Care Medicine, Jinling Hospital, Nanjing University School of Medicine, 305 East Zhongshan Road, Nanjing, Jiangsu 210002, P.R. China

E-mail: yishi201607@163.com

Key words: influenza A, H1N1, non-structural protein 1, interferon, STAT epidemics and occasional pandemic worldwide. Regardless of the great achievements in the modern medicine, the therapeutic strategies for influenza virus infection are not very effective. Annually, influenza viruses cause infection in about $10 \%$ of the human population, and induce more than 250,000 cases of death (1). The reasons for this suboptimal situation are that influenza virus can evade the immune surveillance by antigenic shift and/or altering the microenvironment (2). Therefore, understanding the mechanisms of influenza virus-induced immune evasion is a key to improve the treatments and outcome of influenza virus infection.

Upon influenza virus infection, the innate immune system utilizes germline gene-encoded receptors called pattern recognition receptors (PRRs) to detect the conserved molecular patterns of viruses to initiate antiviral responses, the activation of which results in the production of type I interferon (IFN, mainly IFN $\alpha / \beta$ ), pro-inflammatory cytokines and chemokines (3). Type I IFNs, especially IFN $\beta$, are critical for the clearance of viruses. IFN $\beta$ binds its receptors called IFN $\alpha$ receptor (IFNAR) 1 and IFNAR2 to activate and phosphorylate Janus kinase (JAK)1 and tyrosine kinase (TYK)2. Phosphorylated JAK1 and TYK2 then in turn phosphorylate IFNAR1/2 at specific tyrosine residues in the intracellular part. This phosphorylation leads to the recruitment and the consequent phosphorylation of signal transducers and activators of transcription (STAT)1 and STAT2. A heterodimer formed by phosphorylated STAT1 and STAT2 recruits the IFN-regulatory factor (IFR)9 to generate the IFN-stimulated gene factor (ISGF)3, which results in the production of IFN $\beta$ (4). Thus, STAT1 and STAT2 are essential for the IFN $\beta$-mediated autocrine expression of IFN $\beta$ and the consequent antiviral responses.

Many viruses have the abilities to increase their survival in the host by inhibiting the production of IFNs (5). The mechanisms of virus-induced inhibition of IFNs include disruption of IFN expression, interruption of IFN signaling pathways, and blockage of IFN-associated effectors. The non-structural protein 1 (NS1) of influenza A viruses inhibits the effects of IFN mainly via blocking the expression of IFNs to facilitate influenza virus evasion (6). To the best of our knowledge, NS1 downregulates the expression of IFNs by various mechanisms, 
including: i) Inhibiting the virus detector retinoic acid-inducible gene (RIG-1), the initiator for antiviral responses, which leads to the production of IFN $\beta$ (7); ii) NS1 inhibits the activation of nuclear factor- $\kappa \mathrm{B}(\mathrm{NF}-\kappa \mathrm{B})$ and IRF3 (8), which are required for the production of IFNs (9); and iii) NS1 binds cellular cleavage and polyadenylation specificity factor (CPSF)30 to account for the accumulation of unprocessed mRNAs in the nucleus, thus mRNA production including IFN mRNAs in the cytoplasm is then inhibited (10).

However, the inhibitory roles of NS1 against the IFN production have not been fully understood. It has been reported that NS1 of the laboratory-generated influenza A H1N1 PR/8/34 interacts with RIG-1 to block the production of IFN $\beta$ (11). NS1 of the influenza A H5N1 was demonstrated to inhibit the activation of STAT1 and STAT2 to decrease the expression of IFN $\beta$ (12). However, whether NS1 of the influenza A H1N1 inhibits the production of IFN $\beta$ in human alveolar epithelial cells by affecting the phosphorylation of STAT1 and STAT2 and the loop of IFN $\beta$-mediated autocrine process of IFN $\beta$ production remains elusive. Here, we report that NS1 inhibits the production of IFN $\beta$ in influenza A H1N1 infected alveolar epithelial cells by downregulating the production of IFNAR1 and 2, as well as inhibiting the activation of STAT1 and STAT2.

\section{Materials and methods}

Reagents. Anti-IFN $\beta$ neutralizing antibodies and the corresponding isotype control antibodies (goat IgG control) were purchased from R\&D Systems (Minneapolis, MN, USA). Recombinant human IFN $\beta 1$ 1a (rhIFN $\beta 1$ ) was purchased from PBL Assay Science (Piscataway, NJ, USA). Antibodies for STAT1, p-STAT1, STAT-2, and p-STAT2 were purchased from Cell Signaling Technology, Inc. (Danvers, MA, USA). Fluorescein isothiocyanate (FITC)-conjugated anti-human IFNAR1 antibodies and matched isotype antibodies were from Clinisciences (Nanterre, France). FITC-conjugated anti-IFNAR2 antibodies and isotype antibodies were purchased from Sino Biological Inc. (Beijing, China). Anti-influenza NS1 and anti- $\beta$ actin antibodies were purchased from Santa Cruz Biotechnology, Inc. (Dallas, TX, USA.

Cells and cell culture. The human alveolar epithelial cell line A549 and the Madin-Darby canine kidney (MDCK) cell line were purchased from American Type Culture Collection (Manassas, VA, USA). Cell lines A549 and MDCK were cultured in Dulbecco's Modified Eagle's medium (DMEM) and DMEM: Nutrient Mixture F12 (DMEM/F12, both from Thermo Fisher Scientific, Inc., Waltham, MA, USA), respectively, containing $10 \%$ fetal bovine serum, and $1 \mathrm{X}$ Penicillin-Streptomycin (both from Thermo Fisher Scientific, Inc.) in a humidified incubator with a $5 \% \mathrm{CO}_{2}$ atmosphere at $37^{\circ} \mathrm{C}$.

Virus and cells treated with viral infection. Influenza virus A/Nanjing/1110/2010 (H1N1, referred to as H1N1 below), which lacks NS1 expression, was isolated and cultured. We also isolated and cultured NS1-expressing influenza virus A/Nanjing/1108/2010 (H1N1, referred to as H1N1-NS1 below). The virus culture and storage procedures were approved by local institutions. Influenza virus A H1N1 was grown in 9-day-old embryonated chicken eggs. Virus allantoic fluid (VAF) was collected $48 \mathrm{~h}$ after inoculation, and then titrated with the standard haemagglutination tests (HA) and plaque assays in MDCK cells (13). A549 cells were cultured in 6-cm Petri dishes with the density of $5 \times 10^{6}$ per plate $12 \mathrm{~h}$ before viral infection. A549 cells were infected with H1N1 inocula in VAF at a multiplicity of infection of 1 . Virus inocula were suctioned after $1 \mathrm{~h}$ of co-culture, and then $3 \mathrm{ml}$ of serum-free DMEM medium was added into petri dishes following removing the virus inocula. Infected A549 cells were incubated at $37^{\circ} \mathrm{C}$ and $5 \% \mathrm{CO}_{2}$ for the designed periods.

Virus-infected animal model. Animal experiment protocols were reviewed and approved by the Ethics Committee of Jinling Hospital, Nanjing University School of Medicine (Nanjing, China). Wild C57BL/6 mice (6- to 8-week-old) were purchased from (the Jackson Laboratory, Bar Harbor, ME, USA). Mice were maintained in a specific pathogen-free environment. Mice were infected intratracheally with $1 \times 10^{4}$ plaque forming units (PFU) of influenza A virus under isoflurane anesthesia. Lungs were collected $48 \mathrm{~h}$ post-infection for histological assessment staining (hematoxylin and eosin), flow cytometry, and real-time q-PCR analysis.

Transfection. Cells $\left(2 \times 10^{5}\right)$ were transfected with Lipofectamine LTX (Invitrogen, Carlsbad, CA, USA) following the manufacturer's instruction. Briefly, cells were planted in 6 -well plates $24 \mathrm{~h}$ prior to the transfection procedure. Plasmid DNA or empty vectors and transfection reagents were mixed in serum-free medium and incubated for $30 \mathrm{~min}$ at room temperature. Transfection complexes were added into the corresponding wells of culture plates.

Real-time q-PCR. A549 cells were harvested and used for RNA isolation using a commercial RNeasy mini kit (Qiagen, Valencia, CA, USA). Total RNA $(2 \mu \mathrm{g})$ was reverse-transcribed to cDNA using an RT-PCR kit (Thermo Fisher Scientific, Inc.) according to the manufacturer's instructions. Real-time analysis for IFN $\beta$, IFNAR1, IFNAR2, and control gene HPRT was performed by SYBR-Green Master mix (Thermo Fisher Scientific, Inc.) following the manufacturer's instructions. Relative mRNA expression values were normalized according to a housekeeping gene HPRT. The fold change of mRNA expression was calculated using the formula:

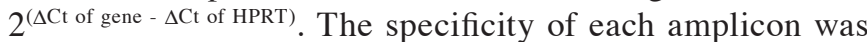
checked by analyzing the corresponding melting curve. The sequences of primers used in real-time PCR were listed in Table I.

Western blot analysis. Whole-cell lysates were obtained in $30 \mu \mathrm{l}$ of lysis buffer (1\% Triton X-100, 0.5\% Nonidet P-40, $10 \mathrm{mM}$ Tris- $\mathrm{HCl}, 150 \mathrm{mM} \mathrm{NaCl}, \mathrm{pH}$ 7.4, $1 \mathrm{mM}$ EDTA, $1 \mathrm{mM}$ EGTA, $0.2 \mathrm{mM}$ phenylmethylsulfonyl fluoride). A total of $50 \mu \mathrm{g}$ of protein was resolved in sodium dodecyl sulfate-polyacrylamide (SDS) gel electrophoresis and transferred to nitrocellulose membranes. Membranes were blocked in 5\% slim milk for $1 \mathrm{~h}$ at room temperature. Then membranes were incubated with the primary antibodies at the optimized titrations at $4^{\circ} \mathrm{C}$ overnight. After incubation with horseradish 
Table I. Primer sequences for quantitative real-time PCR.

\begin{tabular}{ll}
$\begin{array}{l}\text { Target } \\
\text { gene }\end{array}$ & \multicolumn{1}{c}{ Sequence $\left(5^{\prime} \rightarrow 3^{\prime}\right)$} \\
\hline Ifn- $\beta$ & F: TGGGAGGCTTGAATACTGCCTCAA \\
& R: TCCTTGGCCTTCAGGTAATGCAGA \\
Ifnarl & F: CACTGACTGTATATTGTGTGAAAGCCAGAG \\
& R: CATCTATACTGGAAGAAGGTTTAAGTGATG \\
Ifnar2 & F: ATTTCCGGTCCATCTTATCAT \\
& R: ACTGAACAACGTTGTGTTCC \\
HPRT & F: GCAGACTTTGCTTTCCTTGG \\
& R: AAGCAGATGGCCACAGAACT
\end{tabular}

F, forward; R, reverse.

peroxidase-conjugated secondary antibodies, the bands were visualized by using enhancing chemiluminescence system (Amersham Biosciences, Pittsburg, PA, USA). Densitometry analysis was performed with Image $\mathbf{J}$ software.

Flow cytometry. Lungs were collected $48 \mathrm{~h}$ post-infection. Single cell suspensions were obtained by using a commercial lung cell isolation kit (Miltenyi Biotec, Inc., Auburn, CA, USA) according to the manufacturer's instructions. Leukocytes were stained with anti-mouse CD45-APC antibodies (eBioscience, San Diego, CA, USA).

Cells with different treatments were collected by trypsin digestion. Cells were stained with FITC-conjugated anti-human IFNAR1 or INNAR2 antibodies at the designated titrations. Corresponding isotype antibodies were used to indicate the background fluorescence. Cells were applied to an Original Attune Flow cytometer (Applied Biosystems, Foster City, CA, USA). The data were analyzed by Kaluza software 1.3 (Beckman Coulter, Brea, CA, USA).

Statistical analysis. Data were presented as mean \pm SEM (standard error of the means). The difference between two groups was analyzed using a two-tailed student's t-test. The statistical significance between more than two groups was measured by ANOVA followed by Bonferroni's post hoc tests. Statistical analysis was performed by GraphPad Prism 5 Windows Edition (GraphPad Software, La Jolla, CA, USA). A P-value of less than 0.05 was considered statistically significance.

\section{Results}

Mice infected with H1N1-NS1 virus had fewer infiltrated neutrophils and decreased levels of IFN $\beta$ in the lung. First, we checked the effects of H1N1 and H1N1-NS1 viruses in a mouse influenza A infection model, respectively. Mice were intratracheally infected with influenza viruses as described. Forty-eight hours after infection, mice were sacrificed to collect lungs. As demonstrated in Fig. 1A, mice infected with N1N1 had infiltrated leukocytes in lungs, whereas mice treated with H1N1-NS1 viruses showed mild leukocyte infiltration, although histological damage was present. Then, we measured leukocyte infiltration by flow cytometry. The proportion and absolute number of $\mathrm{CD} 45^{+}$leukocytes in the lung were calculated. As shown in Fig. 1B, mice infected with H1N1-NS1 had decreased proportions and numbers of $\mathrm{CD} 45^{+}$leukocytes in the lung, compared to mice treated with $\mathrm{H} 1 \mathrm{~N} 1$, indicating an impaired leukocyte recruitment in the lung of mice infected with NS1-expressing H1N1 viruses. As IFN $\beta$ is a key IFN for the virus defense, we also measured the mRNA levels of IFN $\beta$ in the lung of infected mice (Fig. 1C). Not surprisingly, mice infected with H1N1-NS1 viruses had a lower level of IFN $\beta$ in the lung compared to mice treated with H1N1, suggesting NS1 protein inhibits the production of IFN $\beta$ during influenza $A$ infection. These data show NS1 is an inhibitor for anti-virus immune responses.

A549 cells infected with H1N1 produce IFN $\beta$ via an IFN $\beta$-dependent autocrine mechanism. Human alveolar epithelial cell line A549 was infected with H1N1 (1/64 HA units). Non-infected cells (vehicle-treated) were used as control cells. Forty-eight hours after infection, cells were thoroughly washed by PBS and collected. Purified RNA was obtained using a commercial RNA isolation kit as described in the Materials and methods. The levels of If $n \beta$ mRNA were measured by real-time PCR and normalized to the housekeeping gene HPRT. As shown in Fig. 2A, A549 cells infected with H1N1 had significantly increased levels of If $n \beta$ mRNA compared to non-infected cells. As it has been reported that IFN $\beta$ has autocrine effects on cells producing itself, we questioned whether the upregulation of If $n \beta$ mRNA was an autocrine process. An anti-human IFN $\beta$ neutralizing antibodies or matched isotype control antibodies were administered in culture media. Infected A549 cells treated with anti-IFN $\beta$ neutralizing antibodies $(5 \mu \mathrm{g} / \mathrm{ml})$ in the medium expressed significantly decreased levels of If $n \beta$ mRNA compared to infected cells incubated with isotype control antibodies (Fig. 2B), suggesting IFN $\beta$ itself is involved in this process. Then, we used recombinant human IFN $\beta$ (rhIFN $\beta 1$ ) supplementation (100 IU/ml for $15 \mathrm{~min}$ ) in culture media. As demonstrated in Fig. $2 \mathrm{C}$, hrIFN $\beta 1$ a administration increased If $n \beta$ mRNA levels. These data indicate that cells infected with $\mathrm{H} 1 \mathrm{~N} 1$ produce IFN $\beta$, and IFN $\beta$ acts with its receptors for further expression of IFN $\beta$ via an autocrine mechanism.

NS1 inhibits the production of IFN $\beta$ in A549 cells upon the infection of H1N1. NS1 of influenza A virus exerts antiinflammatory effects to facilitate virus evasion by interfering the IFN $\beta$ signaling pathways (14). However, the precise mechanisms underlying NS1-interferred IFN $\beta$ signaling pathways have not been fully understood. We explored the effects of H1N1 NS1 on IFN $\beta$ production. A549 cells were infected with either H1N1 or H1N1-NS1 viruses. Not surprisingly, A549 cells infected with H1N1-NS1 lost the ability to produce If $n \beta$ mRNA (Fig. 3A). Then, we asked whether NS1 could inhibit IFN $\beta$-mediated IFN $\beta$ production. A549 cells transfected with either empty vectors or those with H1N1 Ns 1 complementary DNA were stimulated with IFN $\beta$ or vehicles. NS1-expressing A549 cells produced lower levels of If $n \beta$ mRNA compared to NS1-null A549 cells in responses to the treatment of hrIFN $\beta 1$ a (Fig. 3B), suggesting NS1 inhibits the autocrine process of IFN $\beta$ expression. 
A

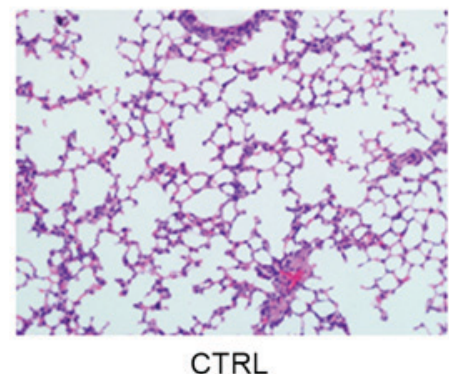

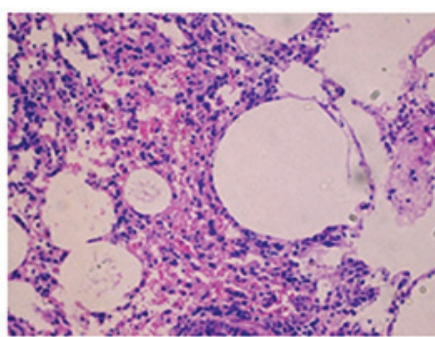

$\mathrm{H} 1 \mathrm{~N} 1$

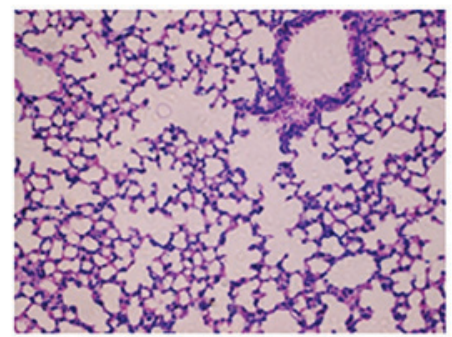

H1N1-NS1

B

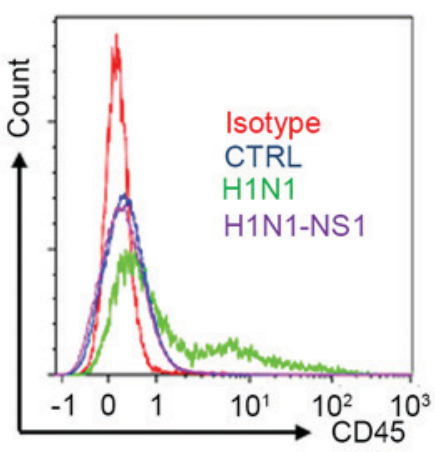

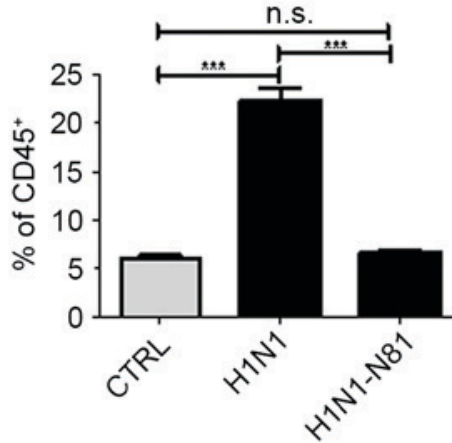

Treatment

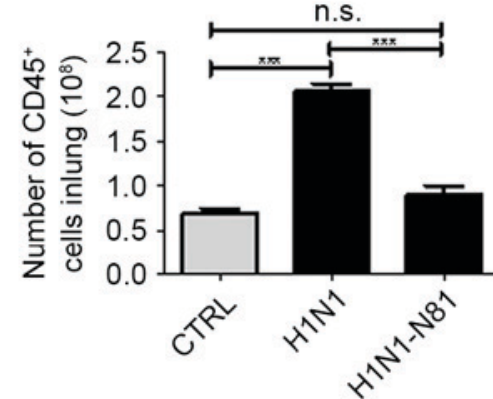

Treatment

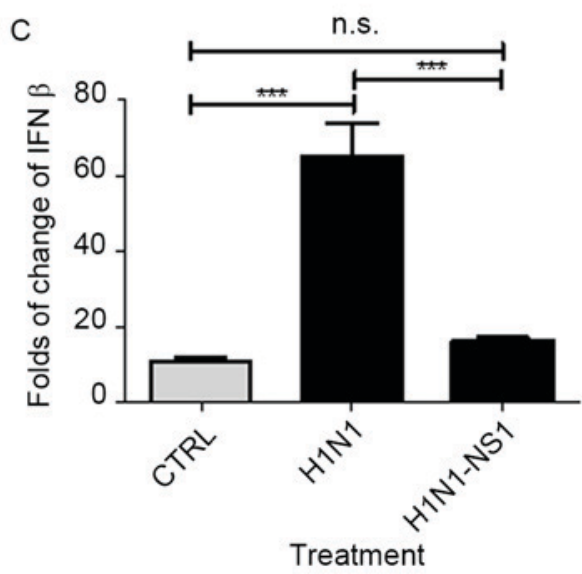

Figure 1. H1N1-NS1 virus induced lung infection harbored fewer immune cells and decreased levels of type I interferons. Lungs were collected from mice $48 \mathrm{~h}$ post-infection. (A) Lung infection induced by H1N1-NS1 showed fewer neutrophil infiltration, although obvious histological damage was present. (B) Mice infected with H1N1-NS1 virus had fewer $\mathrm{CD}^{+} 5^{+}$leukocytes in the lung. Leukocyte infiltration was assessed by the proportion and absolute number of CD45 leukocytes in the lung by flow cytometry. A representative overlay histogram is presented. Mice infected with H1N1-NS1 had a decreased proportion and absolute number of $\mathrm{CD}^{+} 5^{+}$leukocytes infiltrated in the lung post-inflection. (C) Mice infected with N1N1-NS1 had lower levels of IFN $\beta$ in the lung. ${ }^{* * *} \mathrm{P}<0.001$, $\mathrm{n}=6$. NS1, non-structural protein 1; CTRL, control mice treated with intratracheal administration of PBD; n.s., not significant.

NS1 inhibits the upregulation of IFNARI and IFNAR2 upon the infection of H1N1. Next, we explored mechanisms underlying NS1-mediated IFN $\beta$ inhibition. It is possible that NS1 affects many levels of IFN $\beta$ signaling pathways. We firstly examined the effect of NS1 on the receptors of IFN $\beta$, which are IFNAR1 and IFNAR2. A549 cells were transfected with $N s 1$ vectors or empty vectors. Cells were infected with H1N1 24 h after transfection. Cells were then collected $48 \mathrm{~h}$ after the infection process. The expression of Ifnarl and Ifnar2 were assessed by real-time PCR. As shown in Fig. 4A and B, in response to viral infection, both Ifnarl and Ifnar2 mRNA levels were elevated in NS1-lacking cells, however NS1-expressing cells had decreased levels of mRNAs for these two genes. These data suggest the upregulation of receptors for IFN $\beta$ is part of antiviral immunity, and NS1 inhibits this upregulation to build a microenvironment that favors virus survival. The protein levels of IFNAR1 and IFNAR2 were measured using western blot analysis. As demonstrated in Fig. 4C, H1N1 infection increased the expression of IFNAR1 and IFNAR2, while NS1-expressing cells had lower levels of IFNAR1 and IFNAR2, suggesting NS1 inhibits the upregulation of IFNAR1 and IFNAR2 upon viral infection.

We also used flow cytometry to examine the surface expression of IFNAR1 and IFNAR2 of A549 cells, as surface receptors are functional. In parallel to the results of real-time q-PCR and western blot analysis, NS1-harboring A549 cells had a decreased proportion of IFNAR1- and IFNAR2-expressing cells upon the infection of H1N1 compared to cells lacking NS1 (Fig. 4D-F). We infected A549 cells with H1N1 or H1N1-NS1 viruses, and observed similar results as experiments using 
A

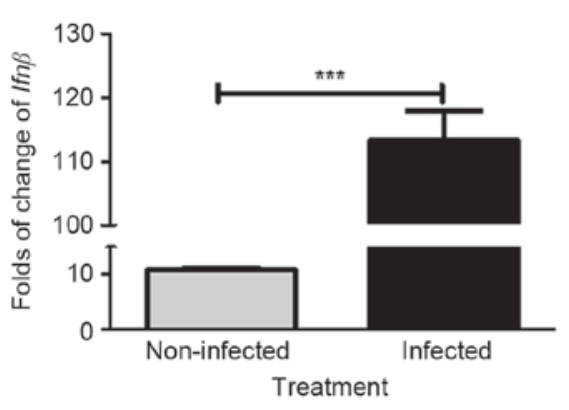

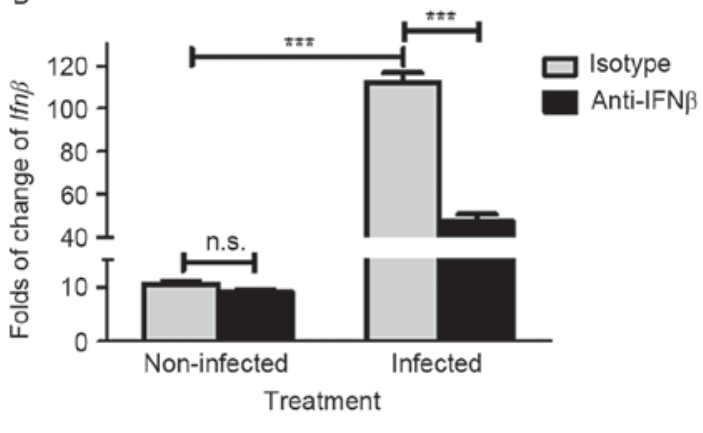

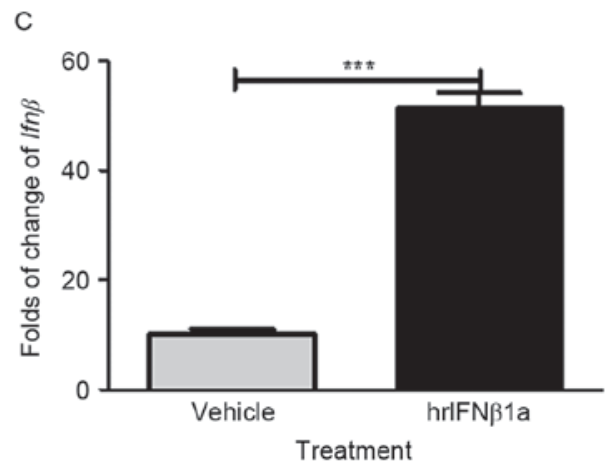

Figure 2. Cultured A549 cells produced IFN $\beta$ upon the infection of influenza A H1N1 in an IFN $\beta$-dependent autocrine fashion. Human alveolar epithelial cell line A549 was cultured in vitro and infected with influenza A. Total RNA was extracted and reverse-transcribed to cDNA. The mRNA expression of IFN $\beta$ was normalized to HPRT after real-time q-PCR. (A) A549 cells infected with H1N1 expressed increased levels of IFN $\beta$ mRNA compared to intact cells (P<0.001, $n=4)$. (B) The expression of IFN $\beta$ of A549 cells infected with H1N1 dependents on IFN $\beta$. The anti-IFN $\beta$ neutralizing antibodies decreased levels of IFN $\beta$ in infected A549 cells $(\mathrm{P}<0.001, \mathrm{n}=4)$. (C) The supplement of hrIFN $\beta 1 \mathrm{a}$ in the culture medium upregulated the expression of IFN $\beta(\mathrm{P}<0.001, \mathrm{n}=4)$. ${ }^{* * *} \mathrm{P}<0.001$. IFN, interferon; n.s., non significant.

A

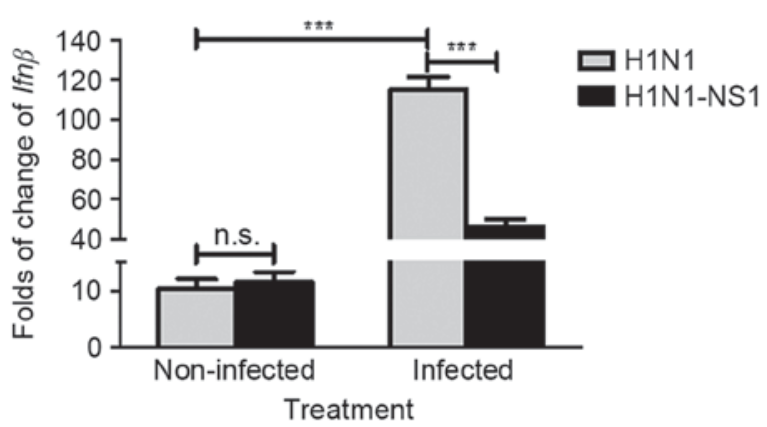

B

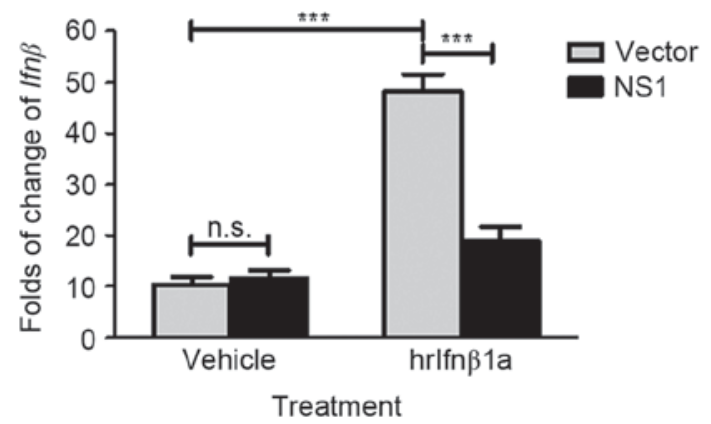

Figure 3. NS1 inhibits the expression of IFN $\beta$ of A5 49 cells infected with H1N1 and treated with exogenous rhIFN $\beta 1$. The expression of IFN $\beta$ was assessed by real-time q-PCR and normalized to the levels of HPRT. (A) A549 cells infected with H1N1-NS1 produced lower levels of IFN $\beta$ compared to cells treated with H1N1 ( $\mathrm{P}<0.001, \mathrm{n}=4$ from one of triplicated experiments). (B) Unlike NS1-null A549 cells, NS1-expressing cells lost the ability to produce IFN $\beta$ upon the treatment of rhIFN $\beta 1 \mathrm{a}\left(\mathrm{P}<0.001, \mathrm{n}=4\right.$ from one of triplicated experiments). ${ }^{* * *} \mathrm{P}<0.001$. NS1, non-structural protein 1 ; IFN, interferon; rhIFN $\beta 1 \mathrm{a}$, recombinant human IFN $\beta 1 \mathrm{a} ;$ n.s., non significant.

transfected cells (data not shown). These data indicate that NS1 inhibits the expression of IFN $\beta$ receptors at both mRNA and protein levels.

NS1 inhibit the phosphorylation of STAT1 and STAT2 to block the production of IFN $\beta$. Then we examined the downstream molecules of IFN $\beta$ receptors. It has been well described that binding between receptors and type I IFNs activates and phosphorylates STAT1 and STAT2 to produce type I IFNs via an autocrine manner $(15,16)$. We assessed the levels of phosphorylated STAT1 and STAT2 in NS1-null and -expressing
A549 cells upon IFN $\beta$ incubation. A549 cells were transfected with vectors of Nsl using the same strategies described above. Cells were incubated with or without the presence of rhIFN $\beta 1 \mathrm{a}$ in media $24 \mathrm{~h}$ after transfection. By the end of incubation, cells were thoroughly washed and collected. Total proteins were obtained as described in Materials and methods. The interested proteins and the corresponding phosphorylated ones were checked by western blot analysis. The representative images of western blot analysis results were shown in Fig. 5. In the steady statues, neither STAT1 nor STAT2 were activated. However, upon the administration of IFN $\beta$ in the medium, 
A

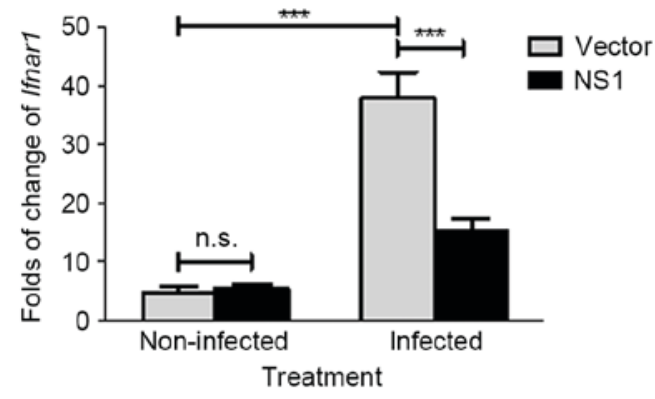

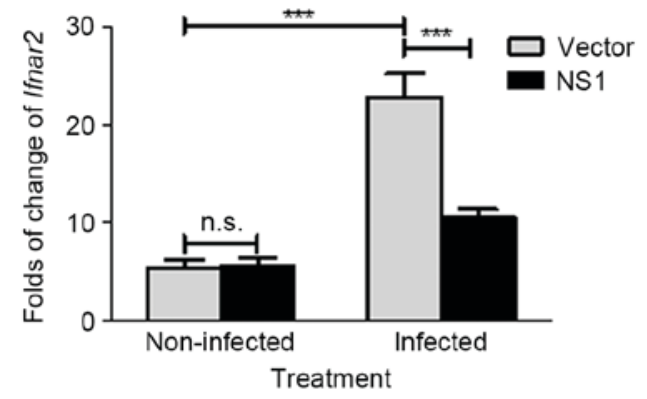

C
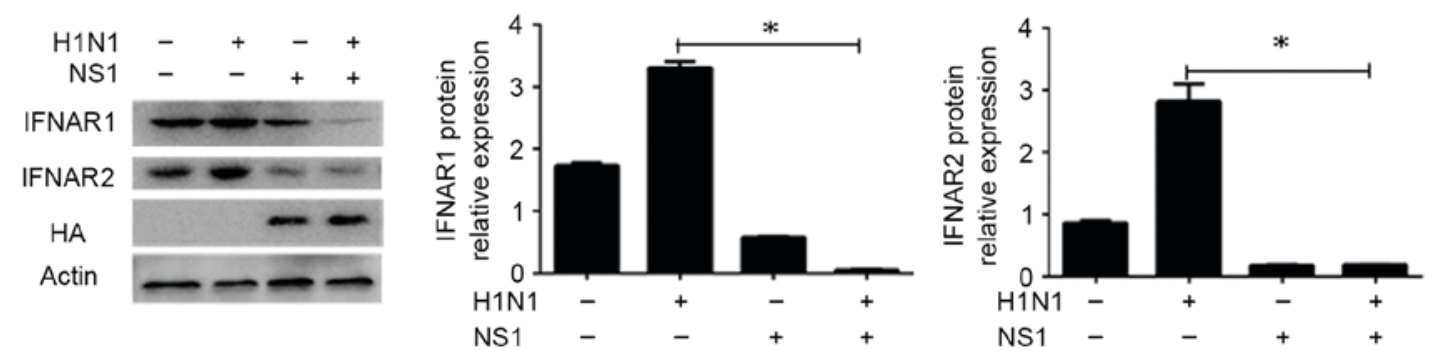

D

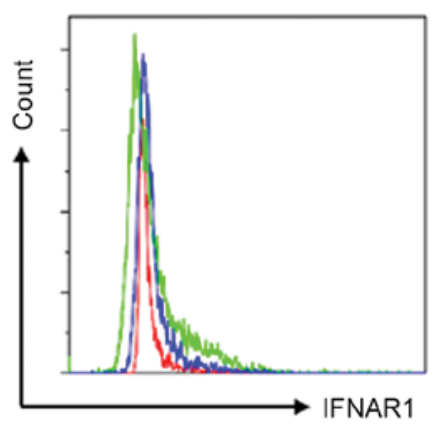

E
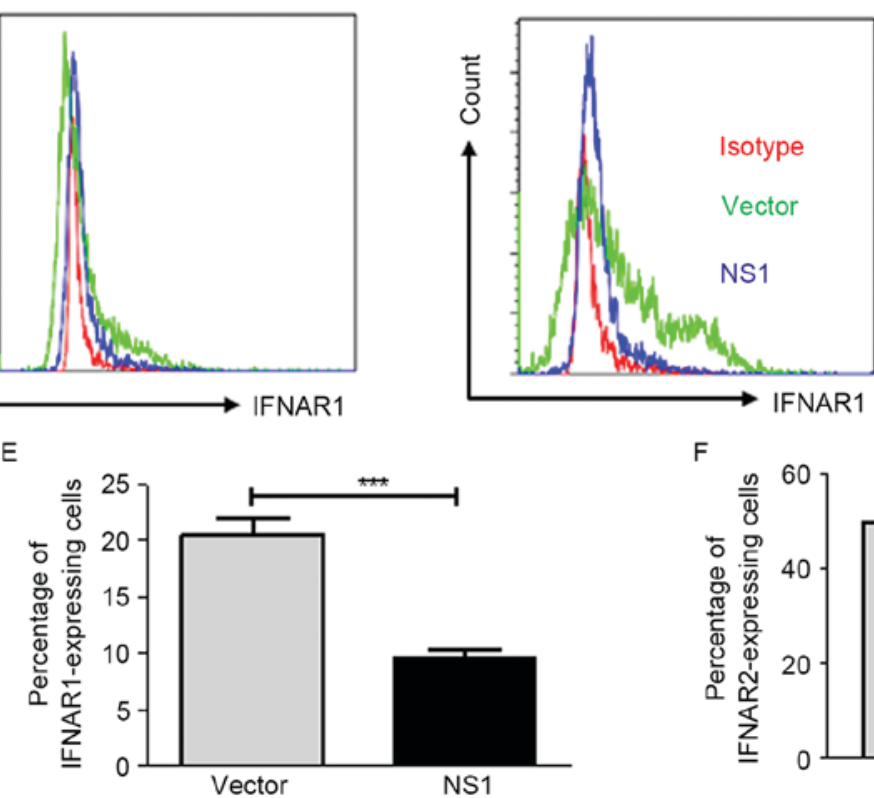

$\mathrm{F}$

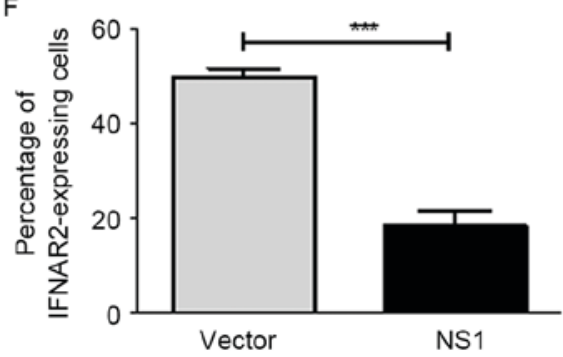

Figure 4. NS1 downregulates the expression of receptors for IFN $\beta$-IFNAR1 and IFNAR2. (A and B) The expression of Ifnarl and Ifnar2 was assessed by real-time q-PCR. NS1-expressing A549 cells produced lower amounts of mRNAs of IFNAR1 and IFNAR2 ( $<20.001, n=4)$. (C) The protein levels of IFNAR1 and IFNAR2 were measured by western blot analysis. NS1 inhibited the upregulation of IFNAR1 and IFNAR2 of A549 cells upon H1N1 infection. (D) The surface expression of IFNAR1 and IFNAR2 on infected A549 cells were checked by flow cytometry. NS1-expressing A549 cells (blue curves) had decreased levels of IFN receptors in A549 cells infected with H1N1. (E) The proportion of IFNAR1-expressing cells of infected A549 cells with or without NS1 expression measured by flow cytometry, $\mathrm{P}<0.001, \mathrm{n}=4$. (F) The percentage of IFNAR2-expressing cells infected with $\mathrm{H} 1 \mathrm{~N} 1, \mathrm{P}<0.001, \mathrm{n}=4 .{ }^{*} \mathrm{P}<0.05$; ${ }^{* * * *} \mathrm{P}<0.001$. NS1, non-structural protein 1; IFN, interferon; HA, haemagglutination tests; n.s., non significant.

NS1-null cells showed strong phosphorylation of STAT1 and STAT2. The strong phosphorylation of STAT1 and STAT2 was decreased in NS1-expressing A549 cells, suggesting NS1 is a negative regulator of the activation of STAT1 and STAT2.

\section{Discussion}

In this study, we demonstrated the effects of H1N1 NS1 on the secretion of IFN $\beta$ in A549 cells upon influenza A H1N1 infection by affecting the production of receptors for IFN $\beta$ and the activation of STAT1 and STAT2 that are essential for IFN $\beta$ secretion. The production of IFN $\beta$ is essential for anti-virus inflammation and clearance. The physiological process of producing IFN $\beta$ is a positive feedback loop via an autocrine mechanism. Upon viral infection, host cells detect the molecular patterns of virus by PRRs and initiate anti-virus responses including the production of IFN $\beta$. IFN $\beta$ itself increases the surface expression of receptors, named IFNAR1 and IFNAR2. IFN $\beta$ then binds IFNAR1 and IFNAR2 to trigger the activation of receptors and the subsequent downstream molecules 

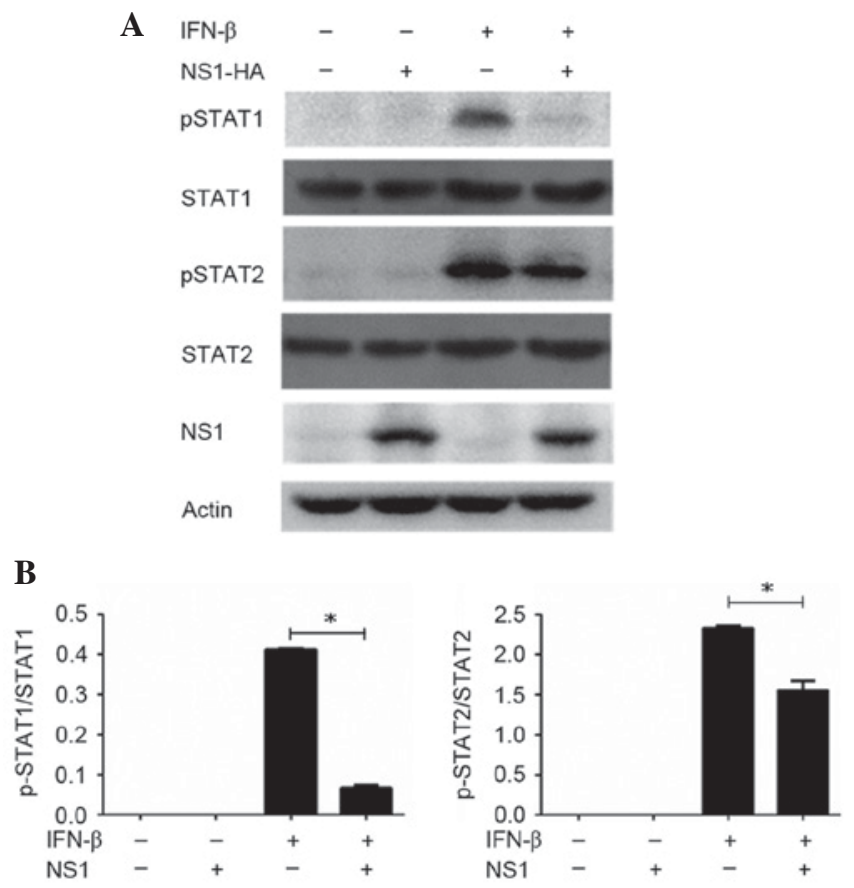

Figure 5. NS1 inhibits the activation of STAT1 and STAT2 of A549 cells upon the treatment of exogenous IFN $\beta$. A549 cells were transfected with empty vectors or vectors containing Ns1. Cells were stimulated with vehicle or hrIFN $\beta 1$ 1a. (A) Representative images of western blot analysis from tripled experiments are demonstrated. (B) IFN $\beta$ increased the levels of phosphorylated STAT1 (pSTAT1) and STAT2 (pSTAT2), which are the active form of STAT1 and STAT2, respectively, in NS1-null cells. However, the increased activation of STAT1 and STAT2 was inhibited in NS1-expressing A549 cells. ${ }^{*} \mathrm{P}<0.05 ; \mathrm{n}=3$ from one of triplicated experiments. NS1, non-structural protein 1; STAT, signal transducer and activator of transcription; IFN, interferon; hrIFN $\beta 1$ a, recombinant human IFN $\beta 1 \mathrm{a}$; HA, haemagglutination tests.

to secret IFN $\beta$. Thus, IFN $\beta$ promotes the production of IFN $\beta$. As it has been reported that influenza A H5N1 NS1 promotes the development of viral evasion (17), in the present study, we tested the hypothesis that NS1 effects IFN $\beta$ production by multiple mechanisms.

It has been reported that A549 cells infected with influenza A viruses bearing NS1 expressed lower levels of type I IFN compared to cells infected with NS1-null virus (18), indicating the effect of NS1 in inhibiting the expression of type I IFN. However, the possible mechanisms of NS1-mediated inhibition of type I IFN production have not been revealed. In the current study, we demonstrated that influenza A H1N1 NS1 protein promotes virus evasion by affecting the production of IFN $\beta$. NS1-expressing alveolar epithelial cell A549 have impaired ability to produce IFN $\beta$ upon the challenge of viral infection or IFN $\beta$, indicating NS1 protein inhibits the $\mathrm{H} 1 \mathrm{~N} 1$-induced IFN $\beta$ production as well as the IFN $\beta$-mediated autocrine secretion of IFN $\beta$. To further analyze the molecular events involved in the NS1-associated inhibition of IFN $\beta$, we checked molecules required in the IFN $\beta$ synthesis process. We observed that NS1 protein downregulates the levels of IFNAR1 and IFNAR 2 to inhibit the binding between IFN $\beta$ and receptors. We also observed that NS1-expressing cells present lower levels of p-STAT1 and p-STAT2 in the existence of IFN $\beta$, which lead to the activation of IRF9 and the following production of IFN $\beta$. These data explain that NS1 protein affects IFN $\beta$ production upon viral infection in the levels of ligand-receptor interaction and activation of transcription factors.

In agreement with previous publications, influenza A NS1 functions as an immune suppressor during responses against viruses with a structure-dependent manner (19). In a study using primary differentiated human tracheobronchial epithelial cells infected with influenza A virus bearing a truncated NS1 mutation (20), results showed lung epithelial cells infected with NS1 mutant virus had less viral replication and more type I IFN production, compared to cells infected with virus having functional NS1. Moreover, dendritic cells infected with NS1 mutant viruses demonstrated increased levels of activation and enhanced abilities to prime naïve T cells with the comparison to dendritic cells incubated with viruses having intact NS1. These data indicate NS1 has an important role to inhibit type I IFN production and dendritic cell activation to favor virus replication.

Type I IFNs are critical for anti-virus responses. Type I IFNs inhibit viral replication and invasion via the production of hundreds of genes including pro-inflammatory cytokines and chemokines that are required for the clearance of virus and virus-infected cells (21). Additionally, Type I IFNs promote the differentiation and maturation of dendritic cells, which present processed viral antigens along with the major histocompatibility complex to effective $\mathrm{T}$ cells. In addition, type I IFNs increase co-stimulatory molecule expression to facilitate the activation of virus-specific $\mathrm{CD} 4^{+}$and $\mathrm{CD} 8^{+} \mathrm{T}$ cells (21).

Influenza A NS1 protein is a series of peptide with the length between 215 and 237 amino acids, which composes of two mainly functional domains: N-terminal RNA-binding domain and C-terminal effector domain (19). NS1 protein favors virus evasion via various mechanisms, among which inhibiting the expression of IFNs is the most important one. It has been observed that NS1 inhibits the activation of IRF3 and $\mathrm{NF}-\kappa \mathrm{B}$ that are needed for the production of IFNs (8). RIG-1 recognizes and binds the PAMP in rival RNA, and subsequently activates the transcription for IFNs (22). NS1 can block the effects of RIG-1 in producing IFN $\beta$ (7). NS1 binds CPSF30 that is required for the 3 ' end processing of pre-mRNA (23). As a result, unprocessed mRNAs accumulate in the nucleus, and the production of mRNAs in cytoplasm is inhibited, including mRNAs for IFNs (10). In the current study, for the first time, we extend the understanding of inhibitory roles of NS1 on IFN production. NS1 can inhibit the production of IFNAR1 and IFNAR2 that are required for the autocrine production of IFNs. NS1 also blocks the activation of STAT1 and STAT2, which are important transcription factors for the expression of IFN $\beta$.

In conclusion, NS1 is an essential player during virus evasion by inhibiting the production of IFN $\beta$ in host cells. In the future, therapeutic strategies targeting NS1 will improve the outcome of influenza A H1N1 infection.

\section{Acknowledgements}

This study was supported by the National Natural Science Foundation of China (grant no. 81470206 to Dr Yi Shi). The funding provider had no roles in the experiment design, data collection and analysis, decision of publication, or preparation of the manuscript. 


\section{References}

1. McCaughey C: Influenza: A virus of our times. Ulster Med J 79: 46-51, 2010.

2. Yan $\mathrm{N}$ and Chen ZN: Intrinsic antiviral immunity. Nat Immunol 13 214-222, 2012

3. Opitz B, van Laak V, Eitel J and Suttorp N: Innate immune recognition in infectious and noninfectious diseases of the lung. Am J Respir Crit Care Med 181: 1294-1309, 2010.

4. Schneider WM, Chevillotte MD and Rice CM: Interferonstimulated genes: A complex web of host defenses. Annu Rev Immunol 32: 513-545, 2014.

5. Bonjardim CA, Ferreira PC and Kroon EG: Interferons: Signaling, antiviral and viral evasion. Immunol Lett 122: 1-11, 2009.

6. Fernandez-Sesma A: The influenza virus NS1 protein: Inhibitor of innate and adaptive immunity. Infect Disord Drug Targets 7: 336-343, 2007.

7. Guo Z, Chen LM, Zeng H, Gomez JA, Plowden J, Fujita T, Katz JM, Donis RO and Sambhara S: NS1 protein of influenza A virus inhibits the function of intracytoplasmic pathogen sensor, RIG-I. Am J Respir Cell Mol Biol 36: 263-269, 2007.

8. Talon J, Horvath CM, Polley R, Basler CF, Muster T, Palese P and García-Sastre A: Activation of interferon regulatory factor 3 is inhibited by the influenza A virus NS1 protein.J Virol 74: 7989-7996, 2000.

9. Mibayashi M, Martínez-Sobrido L, Loo YM, Cárdenas WB, Gale M Jr and García-Sastre A: Inhibition of retinoic acid-inducible gene I-mediated induction of beta interferon by the NS1 protein of influenza A virus. J Virol 81: 514-524, 2007.

10. Das K, Ma LC, Xiao R, Radvansky B, Aramini J, Zhao L, Marklund J, Kuo RL, Twu KY, Arnold E, et al: Structural basis for suppression of a host antiviral response by influenza A virus. Proc Natl Acad Sci USA 105: 13093-13098, 2008.

11. Opitz B, Rejaibi A, Dauber B, Eckhard J, Vinzing M, Schmeck B, Hippenstiel S, Suttorp N and Wolff T: IFNbeta induction by influenza A virus is mediated by RIG-I which is regulated by the viral NS1 protein. Cell Microbiol 9: 930-938, 2007.

12. Jia D, Rahbar R, Chan RW, Lee SM, Chan MC, Wang BX, Baker DP, Sun B, Peiris JS, Nicholls JM and Fish EN: Influenza virus non-structural protein 1 (NS1) disrupts interferon signaling. PLoS One 5: e13927, 2010.
13. Gao W, Sun W, Qu B, Cardona CJ, Powell K, Wegner M, Shi Y and Xing Z: Distinct regulation of host responses by ERK and JNK MAP kinases in swine macrophages infected with pandemic (H1N1) 2009 influenza virus. PLoS One 7: e30328, 2012.

14. Hale BG, Randall RE, Ortín J and Jackson D: The multifunctional NS1 protein of influenza A viruses. J Gen Virol 89: 2359-2376, 2008.

15. O'Shea JJ, Schwartz DM, Villarino AV, Gadina M, McInnes IB and Laurence A: The JAK-STAT pathway: Impact on human disease and therapeutic intervention. Annu Rev Med 66: 311-328, 2015.

16. O'Shea JJ, Holland SM and Staudt LM: JAKs and STATs in immunity, immunodeficiency, and cancer. N Engl J Med 368: 161-170, 2013.

17. Van Hoeven N, Belser JA, Szretter KJ, Zeng H, Staeheli P, Swayne DE, Katz JM and Tumpey TM: Pathogenesis of 1918 pandemic and H5N1 influenza virus infections in a guinea pig model: Antiviral potential of exogenous alpha interferon to reduce virus shedding. J Virol 83: 2851-2861, 2009.

18. McDonald B, Urrutia R, Yipp BG, Jenne CN and Kubes P. Intravascular neutrophil extracellular traps capture bacteria from the bloodstream during sepsis. Cell Host Microbe 12: 324-333, 2012.

19. Carrillo B, Choi JM, Bornholdt ZA, Sankaran B, Rice AP and Prasad BV: The influenza A virus protein NS1 displays structural polymorphism. J Virol 88: 4113-4122, 2014.

20. Aslam R, Speck ER, Kim M, Crow AR, Bang KW, Nestel FP, Ni H, Lazarus AH, Freedman J and Semple JW: Platelet Toll-like receptor expression modulates lipopolysaccharide-induced thrombocytopenia and tumor necrosis factor-alpha production in vivo. Blood 107: 637-641, 2006

21. Davidson S, Maini MK and Wack A: Disease-promoting effects of type I interferons in viral, bacterial, and coinfections. J Interferon Cytokine Res 35: 252-264, 2015.

22. Takeuchi $\mathrm{O}$ and Akira S: Innate immunity to virus infection. Immunol Rev 227: 75-86, 2009.

23. Nemeroff ME, Barabino SM, Li Y, Keller W and Krug RM: Influenza virus NS1 protein interacts with the cellular $30 \mathrm{kDa}$ subunit of CPSF and inhibits 3'end formation of cellular pre-mRNAs. Mol Cell 1: 991-1000, 1998. 